Article

\title{
Concept System and Application of Point Group Symmetry in Mechanical Structure Design
}

\author{
Xiuming Chen * $\mathbb{0}$, Qingying Qiu, Chao Yang and Peien Feng \\ State Key Lab of Computer Aided Design and Computer Graphics, Zhejiang University, \\ Hangzhou 310027, China; medesign@zju.edu.cn (Q.Q.); ycmd@zju.edu.cn (C.Y.); fpe@zju.edu.cn (P.F.) \\ * Correspondence: 11625058@zju.edu.cn
}

Received: 15 August 2020; Accepted: 8 September 2020; Published: 14 September 2020

check for updates

\begin{abstract}
Symmetry has been widely and deeply researched in basic science, and many mature results have been obtained so far. However, the widespread existence of symmetry in applied science is not in direct proportion to the attention it has received. Through a large number of examples studies, almost all mechanical structures are found to have symmetry, and most of them have the characteristics of point group symmetry. Therefore, the concept of point group symmetry in crystallography was extended to the field of machinery and adjusted according to the mechanical structures. First of all, the classification of mechanical point group symmetry is proposed, and how point group symmetry is applied in machinery is illustrated with examples. Then, the requirements of symmetry are analyzed and compared. Furthermore, the data mining software RapidMiner is used to mine the association rules between requirements and symmetry. Based on the mining results, the four selection principles of point group symmetry are summarized to provide ideas for structure design. Finally, a new type of gear pump with radial force balancing is invented by comprehensively using the mining results and selection principles.
\end{abstract}

Keywords: point group symmetry; mechanical structure design; requirements; data mining; selection principles

\section{Introduction}

Symmetry initially referred to balance, harmony, and coordination. Until modern times, generalized symmetry was defined as invariance to change [1]. Physics interprets symmetry as regularity, while aesthetics emphasizes that symmetry is the characteristic of beauty and art [2]. Studies have been conducted on symmetry in the natural science [3-5], social science [6], and engineering science [7]. Landscapes, buildings, and mechanical equipment all show symmetrical attributes.

The study of symmetry is usually inseparable from group theory. In the 1930s, as soon as the group theory came out, the concept of symmetry began to merge with it. After that, group theory has become a mathematical language to describe symmetry in various disciplines [8-10]. But different disciplines could adjust the definition and scope of symmetry group according to their own characteristics. In chemistry, point group and space group have been widely recognized and applied, but the third group between the two groups can still be defined according to specific research object, such as unit cell in solid [11]. In biology, combined with the term biological symmetry, group theory can be used to describe biological objects, such as point group symmetry to describe the shape of petals [12]. In architecture, symmetry of each level, such as point symmetry, rotational symmetry, and translational symmetry, is considered as a mathematical symmetry group. Symmetry group or hierarchical symmetry classification of nested control is helpful to further analyze the function of symmetry or asymmetry [13]. In mechanics, group theory can also be used as a reference for classification [14]. 
Engineering design points out that symmetrical structures satisfy the design criteria of simplicity [15]. Axiomatic design gives a reasoning: as long as FRs (functional requirements) can be met, symmetrical shapes or components should be used as far as possible [16]. The principle of increasing asymmetry in TRIZ (invention problem solving method) emphasizes the innovative idea of symmetry breaking [17]. The earliest systematic research on mechanical symmetry is the applied knowledge of mechanical structure symmetry summarized by Barenschen in his doctoral dissertation [18]. Other common research studies on mechanical symmetry include symmetry recognition or detection, the influence of symmetry features on DFA (design for assembly), the use of symmetry ideas to reduce the calculation time or modeling difficulty [19-21], etc. There are also some scattered research studies on mechanical symmetry, such as using symmetry to restore man-made objects layer by layer, using symmetry as a dynamic feature to solve problems in artificial vision, taking symmetry as one of the criteria for structural optimization [22-25], etc. In a word, mechanical symmetry, like other fields, is usually a method or tool to guide the application.

The relationship between symmetry and requirement is the key point of symmetrical structure design. For example, the point group symmetry is applied to the classification of mechanical parts to study the relationship between the symmetry and assembly efficiency [26], and the idea of symmetry breaking can be used in error proofing design [27].

In view of the advantages of group theory to symmetry classification and the previous achievements, this paper starts with point group symmetry and focuses on its concept and application in mechanical structure.

\section{Method}

Association rules mining can discover the association between items from the data set. Through the analysis of a large number of examples, we can get the data set which takes point group symmetry and requirements as the items. Then, we can use the data analysis software RapidMiner to mine the association rules between point group symmetry and requirements.

\subsection{Data Preparation}

\subsubsection{Classification of Point Group Symmetry}

In crystallography, the various combinations of all symmetry elements converging at one point is called the point group, or symmetry type. The mathematical analysis shows that there are 32 kinds of spatial point groups in crystal [28]. Based on the crystallographic definition of point group symmetry and the Schoenflies symbol, the point group symmetry of mechanical structures can be classified as uniaxial group symmetry, nonaxial group symmetry, and multiaxial group symmetry, according to the number of main axes. According to the types and combinations of symmetry elements, uniaxial group symmetry is divided into 7 kinds, uniaxial group symmetry is divided into 2 kinds, and multiaxial group symmetry is not divided in detail. The concept system of point group symmetry of mechanical structures can be obtained as shown in Figure 1.

The symbol and symmetry elements of point group symmetry are shown in Table 1 . The value of $n$ is no less than 2 in all symbols. 


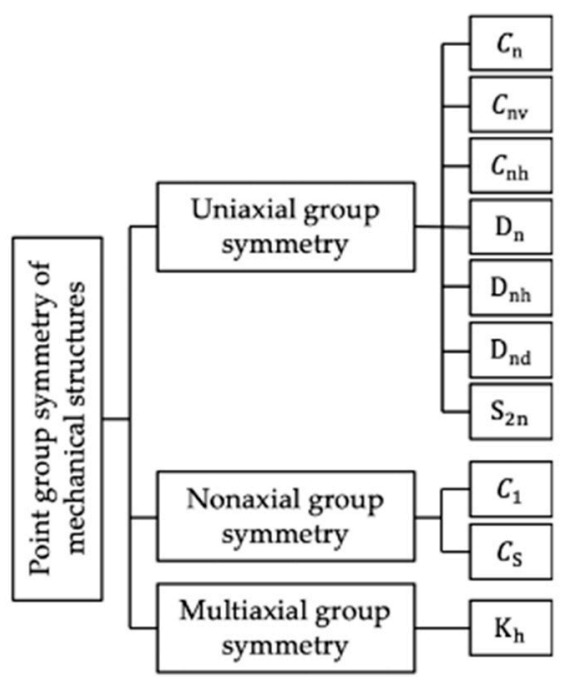

Figure 1. Classification of point group symmetry.

Table 1. Symbols and symmetry elements of point group symmetry.

\begin{tabular}{ccc}
\hline Symbols & Symmetry Elements & The Relationship between Symmetry Elements \\
\hline$C_{1}$ & $C_{1}$ & \\
$C_{S}$ & $\sigma$ & $C_{n}$ \\
$C_{n}$ & $C_{n}, \sigma_{v}$ & \\
$C_{n v}$ & $C_{n}, \sigma_{h}$ & $\sigma_{h}$ passes through $C_{n}$, the number of $\sigma_{v}$ is $n$ \\
$C_{n h}$ & $C_{n}, C_{2}$ & $C_{2}$ is perpendicular to $C_{n}$, the number of $C_{2}$ is $n$ \\
$D_{n}$ & $C_{n}, C_{2}, \sigma_{h}$ & $/$ \\
$D_{n h}$ & $C_{n}, C_{2}, \sigma_{d}$ & $\sigma_{d}$ passes through $C_{n}$ and bisects the angle between $C_{2}$ \\
$D_{n d}$ & $C_{n}, C_{2 n} \sigma_{h}$ & $C_{2 n} \sigma_{h}$ is the combination of $C_{2 n}$ and $\sigma_{h}$ \\
$S_{2 n}$ & $C_{n}, C_{n}, \ldots, C_{n}$ & multiple $C_{n}$ intersect at one point \\
$K_{h}$ & &
\end{tabular}

\section{(1) Uniaxial group symmetry}

Uniaxial group symmetry means that there is only one main axis in the structure, and main axis refers to the rotational symmetry element with the highest order. The curve ribs shown in Figure $2 \mathrm{a}$ are rotationally symmetrical around the main axis, belonging to $C_{n}$ (in this case, $n=5$ ). In addition to rotational symmetry around the main axis, there are also mirror symmetry elements passing through the main axis, so the straight ribs shown in Figure $2 \mathrm{~b}$ belong to $C_{n v}$, and the number of mirror symmetry elements is equal to the order of rotational symmetry, that is, the number of ribs. The curve shape is more difficult to process than the straight shape, while the even number of straight ribs are deformed by internal stress during the casting process and are also prone to vibration during driving [29]. Therefore, considering the machining difficulty and driving stability, ribs of $C_{(2 n+1) v}$ are generally used in a wheel.

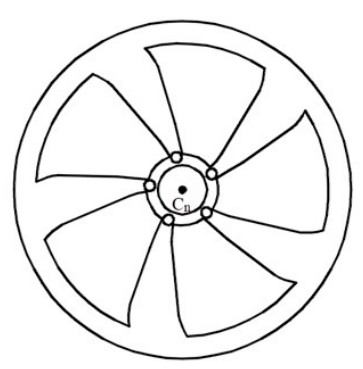

(a)

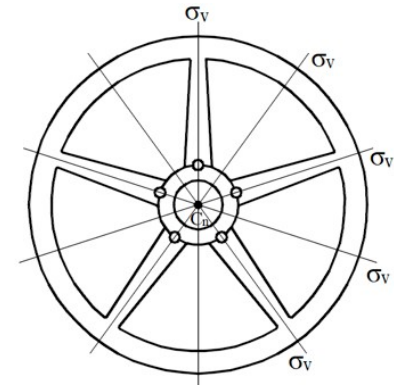

(b)

Figure 2. Wheel ribs. (a) Curved ribs; (b) straight ribs. 
Structure is the carrier of function, and the requirement is the generalized function, including function, performance and constraint. Multiple symmetry elements structure is more widely used in practice than single symmetry element structure because it can not only achieve functions but also improve performance and meet constraints. Impellers in Figure 3 can properly prove this conclusion. The symmetry element of single impeller shown in Figure $3 \mathrm{a}$ is only $C_{n}$, which makes the centrifugal pump produce axial unbalanced force during operation, resulting in vibration and noise. In order to solve this problem, as shown in Figure $3 \mathrm{~b}, \sigma_{h}$ is added to change single impeller into bilateral impeller, and then self-balance is realized [30].

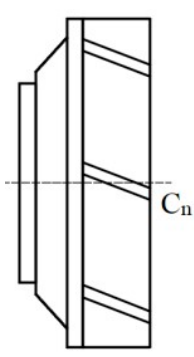

(a)

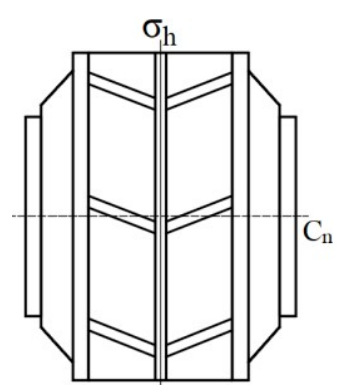

(b)

Figure 3. Impellers. (a) Single impeller $\left(C_{n}\right)$; (b) bilateral impeller $\left(C_{n h}\right)$.

(2) Nonaxial group symmetry

Nonaxial group symmetry includes $C_{1}$ and $C_{S}$. In $C_{1}$, symmetry elements only have identity operation, so both asymmetrical structures and symmetrical broken structures belong to $C_{1}$. $C_{S}$ is also mirror symmetry, with only one mirror surface or line as symmetry element.

There is a principle of increasing asymmetry in TRIZ theory, that is, increasing the asymmetry of asymmetrical structure or changing the symmetrical structure to asymmetrical structure. However, in the comparative analysis of uniaxial group symmetry, it is found that the structures with higher symmetry tend to have better performance, so the principle of increasing asymmetry is not always effective, but it is effective in the process of changing uniaxial group symmetry into nonaxial group symmetry.

In Figure 4, the bearing housing is planned to adopt $C_{n}$ and $C_{S}$ structures, respectively. $C_{n}$ shown in Figure $4 \mathrm{a}$ is easy to be confused in front and back positions, so that the deviation between the axis of the housing hole and the axis of the shaft increases due to the reversal of the front and back positions. However, $C_{S}$ shown in Figure $4 \mathrm{~b}$ can eliminate the deviation, and the bearing housing changes from uniaxial group symmetry to nonaxial group symmetry, which conforms to the principle of increasing asymmetry.

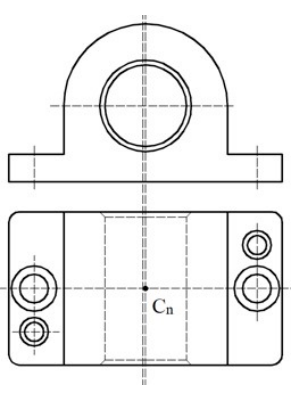

(a)

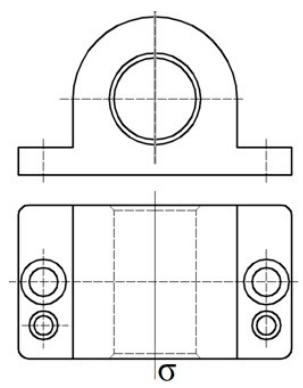

(b)

Figure 4. Bearing housing. (a) Unreasonable design $\left(C_{n}\right)$; (b) improved design $\left(C_{S}\right)$. 


\section{(3) Multiaxial group symmetry}

Multiaxial group symmetry is a general designation for all the point group symmetry with more than one main axis, denoted as $K_{h}$. The most common requirements of multiaxial group symmetry are to expand the scope of function and safety redundancy. The device shown in Figure 5 has three main axes, which can collect vibration energy in multiple directions, that is, expand the scope of function. When the energy collection module is damaged, it can be easily disassembled and replaced. The waste of resources caused by the overall scrap of the energy collector can be reduced due to safety redundancy.

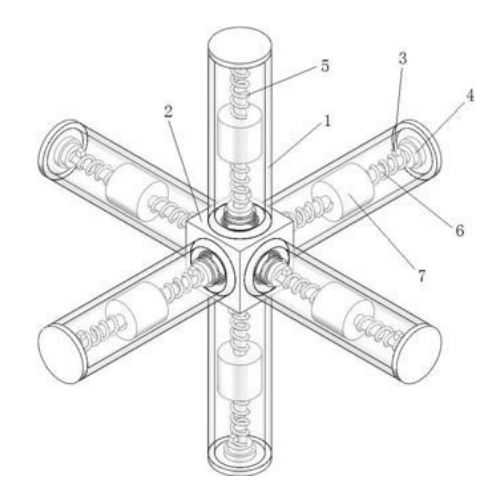

Figure 5. A vibration energy collecting device [31].

\subsubsection{The Analysis of Requirements}

In addition to the examples listed above, the realization of functions and the improvement of performance by point group symmetry is reflected in many aspects. In the process of analyzing the relationship between point group symmetry and requirements, requirements are divided into functions and performance in this paper. Structure design should first meet functions and subsequently consider specific performance. Symmetry element $C_{n}$ is usually used to achieve main functions, and other symmetry elements besides $C_{n}$ are usually used to improve performance. This shows that increasing symmetry is a way to improve structural performance. For example, different types of gears all have symmetry element $C_{n}$ to meet the transfer of power and motion, but symmetry element $\sigma_{h}$ in spur gear and herringbone gear counteracts side effect, and symmetry element $\sigma_{v}$ in spur gear and bevel gear puts emphasis on reducing the difficulty of processing.

Based on the analysis of mechanical structures, the requirements of symmetry elements of point group symmetry are summarized, as shown in Table 2.

Combining the contents of Tables 1 and 2, the requirements corresponding to the point group symmetry can be found. Take $K_{h}$ as an example, $K_{h}$ contains multiple symmetry elements $C_{n}$, so $K_{h}$ can not only meet all the requirements $C_{n}$ meets but also repeat these requirements many times. The repetition of requirements can be expressed as expanding the scope of functions, increasing the efficiency of functions, as well as increasing the intensity of functions and safety redundancy. $K_{h}$ and its requirements are shown in Table 3. The requirements satisfied by all the other types of point group symmetry can be analogized in turn, which will not be described in detail here. As for nonaxial group symmetry $C_{S}$, its symmetry element is only $\sigma$, and the corresponding requirements include all the requirements that $\sigma_{v}, \sigma_{h}$, and $\sigma_{d}$ can meet. 
Table 2. Symmetry elements and their requirements.

\begin{tabular}{|c|c|c|c|c|}
\hline \multirow{2}{*}{ Requirements } & \multicolumn{4}{|c|}{ Symmetry Elements } \\
\hline & \multicolumn{2}{|c|}{$C_{n}$} & \multicolumn{2}{|c|}{$\sigma_{v}$} \\
\hline Functions & $\begin{array}{r}\text { Transform } \\
\text { Control pow } \\
\text { Transfer pon } \\
\text { Join or sep } \\
\text { Change the sl } \\
\text { Store } \\
\text { Fixate or move the }\end{array}$ & $\begin{array}{l}\text { ergy types } \\
\text { and motion } \\
\text { and motion } \\
\text { te material } \\
\text { pe of material } \\
\text { terial } \\
\text { osition of material }\end{array}$ & & \\
\hline Performance & \multicolumn{2}{|c|}{$\begin{array}{l}\text { Expand the scope of function } \\
\text { Counteract side effects }\end{array}$} & \multicolumn{2}{|c|}{$\begin{array}{c}\text { Reduce the difficulty of processing } \\
\text { Reduce friction coefficient } \\
\text { Reduce volume } \\
\text { Disassemble easily }\end{array}$} \\
\hline \multirow[b]{2}{*}{$\begin{array}{l}\text { Requirements } \\
\text { Functions }\end{array}$} & \multicolumn{4}{|c|}{ Symmetry Elements } \\
\hline & \multicolumn{2}{|r|}{$C_{2}$} & $\sigma_{d}$ & $S_{2 n}$ \\
\hline Performance & $\begin{array}{c}\text { Expand the scope } \\
\text { of function } \\
\text { Reduce volume } \\
\text { Counteract side } \\
\text { effects }\end{array}$ & $\begin{array}{c}\text { Reduce area } \\
\text { Extend service life } \\
\text { Reduce vibration } \\
\text { and shock } \\
\text { Increase stability } \\
\text { Reduce } \\
\text { deformation }\end{array}$ & $\begin{array}{c}\text { Eliminate } \\
\text { interference }\end{array}$ & $\begin{array}{l}\text { Counteract side } \\
\text { effects } \\
\text { Reduce vibration } \\
\text { and noise }\end{array}$ \\
\hline
\end{tabular}

Table 3. Requirements that $K_{h}$ can meet.

\begin{tabular}{ccc}
\hline \multicolumn{2}{c}{ Functions } & Performance \\
\cline { 2 - 3 } & $\begin{array}{c}\text { Transform energy types } \\
\text { Control power and motion } \\
\text { Transfer power and motion } \\
\text { Join or separate material } \\
\text { Change the shape of material } \\
\text { Store material }\end{array}$ & $\begin{array}{c}\text { Expand the scope of function } \\
\text { Improve the efficiency of function } \\
\text { Increase the intensity of function } \\
\text { Counteract side effects } \\
\text { Safety redundancy }\end{array}$ \\
Fixate or move the position of material & \\
\hline
\end{tabular}

It can be seen from Tables 2 and 3 that a requirement may be realized by several symmetry types. Through association rules mining, it is possible to compare the association strength between point group symmetry and requirements and provide a reference for structural schemes selection.

\subsection{Association Rules Mining}

This paper used RapidMiner to mine association rules. RapidMiner is an open source data analysis tool that connects the data mining process by dragging operators. Each operator is a process or an algorithm. In order to mine the association rules between point group symmetry and requirements, we only need to drag operators and connect them to establish the model.

\subsubsection{Data Preprocessing}

After analyzing the symmetry type and requirement of mechanical structures (such as the examples in Figures 2-4, etc.), the symmetry type and requirement are regarded as the items and are organized into a file format that can be recognized by RapidMiner. The sample before and after the file conversion is shown in Figure 6. 
(a)

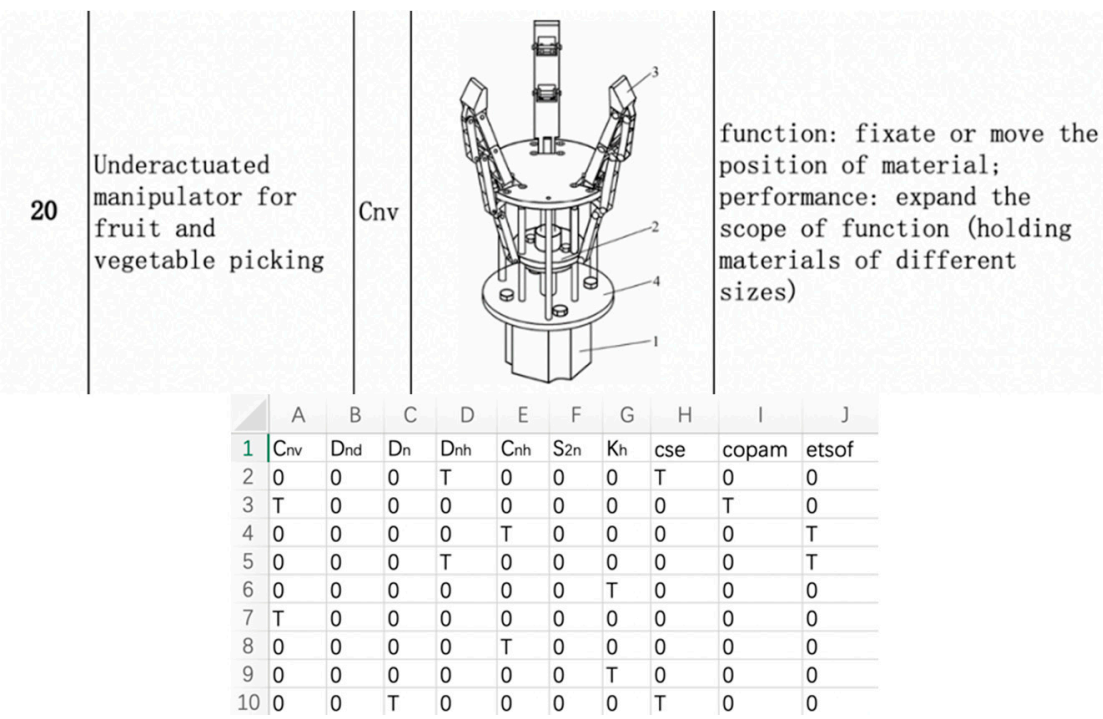

Figure 6. File sample. (a) Sample of structure analysis; (b) sample of files read by RapidMiner.

\subsubsection{Data Mining Modeling}

(1) Drag the "Read Excel" operator to read the data.

(2) Drag the "Nominal to Binominal" and "Select Attributes" operators to solve the problem of semantic deviation and retain the items with semantic existence.

(3) Drag the "FP-Growth" and "Create Association Rules" operators and connect all operators to complete the modeling. The final association rules mining model is shown in Figure 7.

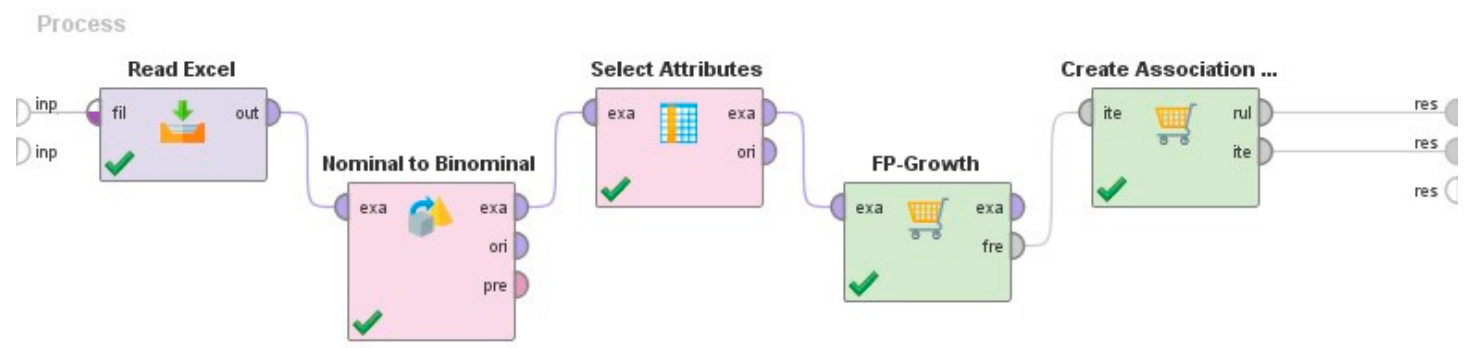

Figure 7. Association rules mining model.

\section{Results}

\subsection{Mining Results}

Run the model shown in Figure 7 to get the results of association rules mining. The rule strength is mainly measured by support and confidence. Support means the proportion of an item in the data set. Confidence is a kind of conditional probability, which reflects the proportion of conclusion (symmetry type) when premise (requirement) appears [32]. The following is an analysis of association rules with high support and high confidence.

Figure 8 shows single items with support more than $8 \%$. In point group symmetry, $D_{n h}$ has the highest support (34.7\%), followed by $C_{n v}(19 \%)$. In the requirements, "transfer power and motion (topam)" has the highest support (10.9\%), followed by "expand the scope of function (etsof)" (8.4\%).

Set the minimum confidence to $25 \%$. Taking $D_{n h}$ as an example, there are 12 premises to get $D_{n h}$, as shown in Figure 9. The requirements with top three support are "topam", "cse", and "etsof", as shown in Figure 8. All these three requirements can be taken as the premise to get $D_{n h}$. Among them, the support and confidence obtained by associating "topam" with $D_{n h}$ are the highest, which are 6.8 and $62.2 \%$, respectively. As for "cse", the support and confidence associated with $D_{n h}$ are higher than 
those associated with $C_{n v}$, as shown by No. 47 in Figure 9 and No. 30 in Figure 10. This comparison also provides a reference for later selection principles and structure design.

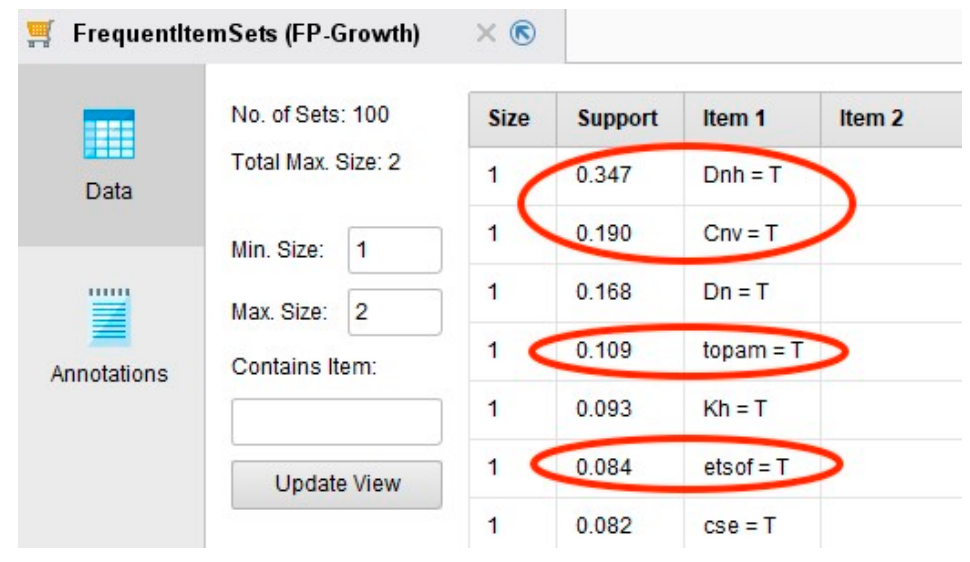

Figure 8. Support of high frequency items.

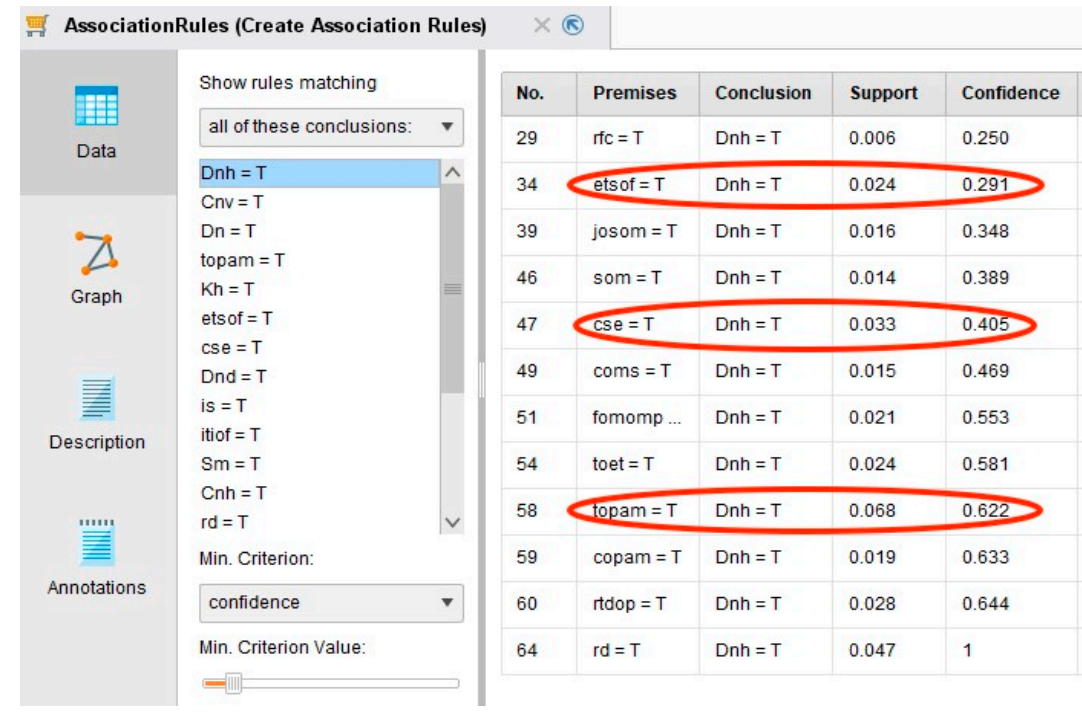

Figure 9. Association rules about $D_{n h}$.

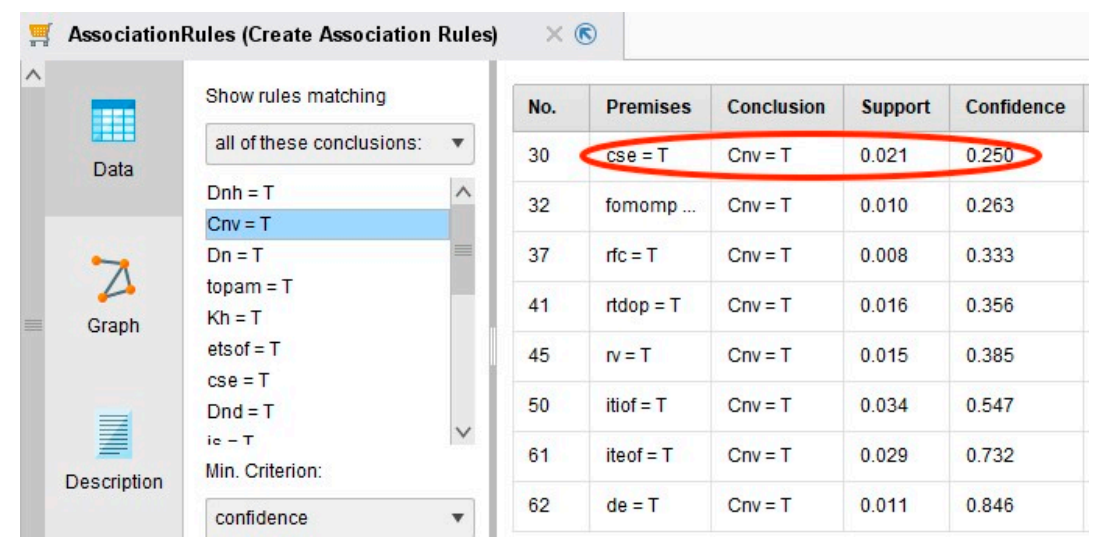

Figure 10. Association rules about $C_{n v}$.

By analyzing the results mined by association rules, several general selection principles can be summarized. In this way, it is possible to use requirements as the premise and refer to the support and confidence to obtain the appropriate symmetrical structure solution. 


\subsection{Selection Principles}

In the design process, if the requirements that match the selection principles are met, there is no need to find possible solutions from a large number of mining results, thereby saving time and reducing errors.

\subsubsection{Principle 1: Increase Symmetry}

Usually, we have consensus that a circle is more symmetrical than a square and that a square is more symmetrical than a rectangle. The influencing factors of symmetry are various. Thus far, no quantitative comparison is available of symmetry between two different structures. Figure 11 shows the qualitative comparison of point group symmetry. It can be seen that the highest symmetry is the multiaxial group, and the lowest is the nonaxial group. In uniaxial group symmetry, $C_{n}$ has the lowest symmetry, and $D_{n h}$ has the highest symmetry.

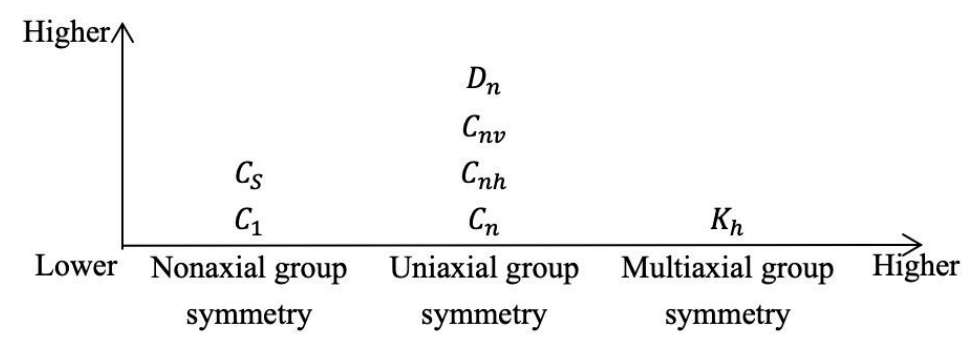

Figure 11. Qualitative comparison of symmetry.

Simplicity, safety, and clarity are the design criteria [15]. Structures with higher symmetry satisfy the simplicity criterion of design. For example, the spur gear of $D_{n h}$ is simpler to manufacture than other gears. Compared with other uniaxial group symmetry, $D_{n h}$ increases symmetry by increasing the spatial dimensions of symmetry elements, thereby improving the performance of the structures. As long as there is no reason for asymmetry, the structural scheme with higher symmetry shall be adopted as far as possible.

From the data mining results shown in Figure 8, we can also see that the support of $D_{n h}, C_{n v}, D_{n}$, and $K_{h}$ are $34.7,19,16.8$, and $9.3 \%$, respectively, ranking in the top four of all point group symmetry, indicating that in practical applications, point group structures with high symmetry are more applied, which conforms to the principle of increasing symmetry.

Figure 12 is a case of applying selection principle 1 to improve the structure. The traditional electric fan belongs to $C_{n}$ as shown in Figure 12a, and the order of rotational symmetry is three, which is the same as the number of fan blades, but it can usually be five or other odd numbers. The odd order can reduce vibration and noise caused by fan operation. The structure of $C_{n}$ can only produce wind on the front side of the blades. To meet the requirement of one fan for continuous use by additional people, a Korean design company has introduced a double-sided fan, which known as the "2-way" fan. As shown in Figure 12b, the new fan belongs to $D_{n}$. The order of main axis is even, which reduces the difficulty of assembling blades. Moreover, because of the symmetry element $C_{2}$, the noise problem caused by even order is solved. The structural design of $D_{n}$ can produce wind from front and back, while the swing angle of the traditional electric fan is approximately $45^{\circ}$ to the left or right. Compared with $C_{n}, D_{n}$ breaks the limitation of wind outlet from front side, which means expanding the scope of the function. 


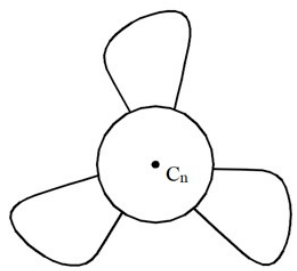

(a)

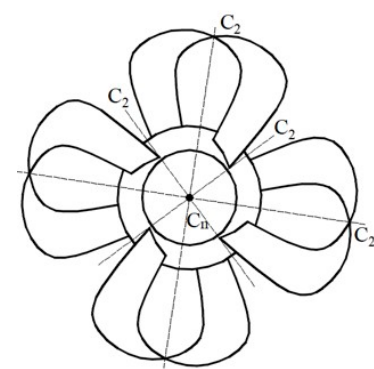

(b)

Figure 12. Electric fan. (a) Traditional fan; (b) "2-way" fan.

\subsubsection{Principle 2: Increase Asymmetry}

For a specific design requirement, if there are multiple schemes of point group symmetry that can meet it, the scheme with higher symmetry should be preferred, no matter from the perspective of simplicity, safety or aesthetics. Therefore, increasing symmetry is the normal design thinking, and increasing asymmetry only occurs under the condition of restrictions.

Changing a multiaxial group symmetry into a uniaxial group symmetry, especially a uniaxial group symmetry into a nonaxial group symmetry, is a way to increase asymmetry, which is often used in error proofing design. The improvement of the bearing housing shown in Figure 4 is a typical case.

The result of asymmetry often has the cause of asymmetry, so another important factor of increasing asymmetry is the constraint of asymmetry.

Lifting building components needs to ensure the stability of the process. For building components with asymmetrical internal structures, asymmetrical lifting traverse is needed. For asymmetrical building components, if the mirror-symmetrical lifting traverse shown in Figure 13a is used for lifting, the geometrical center line of the lifting appliance cannot coincide with the geometrical center line of the lifted object. During the lifting process, the object to be lifted rocking and tilting will occur. Under the constraints of the asymmetry of the building components, it is necessary to increase asymmetry of the lifting traverse. For the symmetry breaking lifting traverse shown in Figure 13b, the bracket can be added, and the position of the lifting lugs can be changed, according to the actual structure of the lifted object to keep asymmetrical objects in balance during lifting.

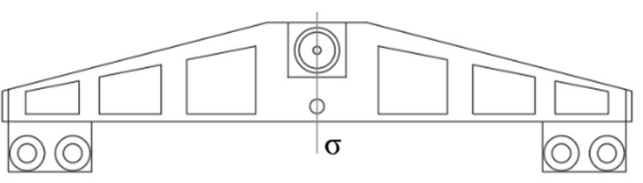

(a)

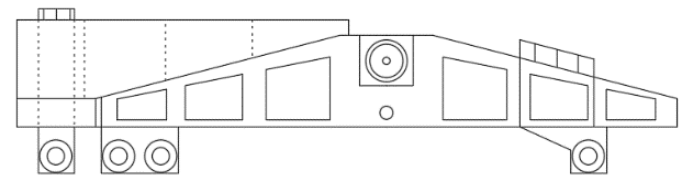

(b)

Figure 13. Lifting traverse [33]. (a) Unreasonable design $\left(C_{S}\right)$; (b) improved design $\left(C_{1}\right)$.

\subsubsection{Principle 3: Priority}

As shown in the results of data mining in Figure 8, the number of practical applications of $D_{n h}$ and $C_{n v}$ is more than that of the other types, combined with selection principle 1 , when the requirements can be satisfied by all point group symmetry, $D_{n h}$ is the first priority, and $C_{n v}$ is the next.

$D_{n h}$ cannot appear in two-dimensional graphs. As shown in Figure 14, by increasing the number of keyways, the shaft section can be changed from $C_{S}$ to $C_{n v}$, which can reduce the deformation of the shaft caused by stress concentration, thus reducing the rejection rate. Similar common application example is H-shaped long beam, as shown in Figure 15, the $C_{n v}$ section avoids bending deformation of long beam. 


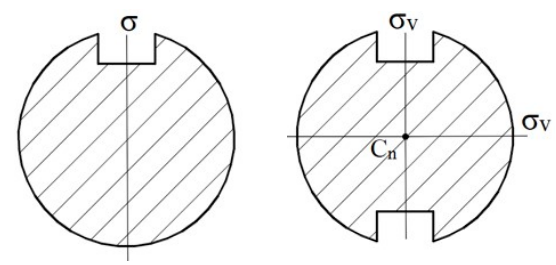

Figure 14. Shaft sections.

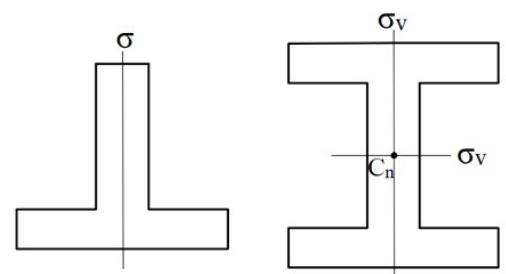

Figure 15. Beam sections.

Although the support of $K_{h}$ is also ranked the top four, its structure is more complicated than the uniaxial group symmetry, so $K_{h}$ is a priority only when the requirement is to expand the scope of the function.

The support of $D_{n d}$ and $S_{2 n}$ is the smallest as shown in Figure 16, indicating that $D_{n d}$ and $S_{2 n}$ have the least priority in practice, usually as an improvement plan of $D_{n h}$ and $C_{n h}$. For example, a self-aligning roller bearing of $D_{n d}$ can adjust to the angular deviation between the inner and outer raceways. Herringbone tires of $S_{2 n}$ have better anti-skid performance and pollution discharge performance.

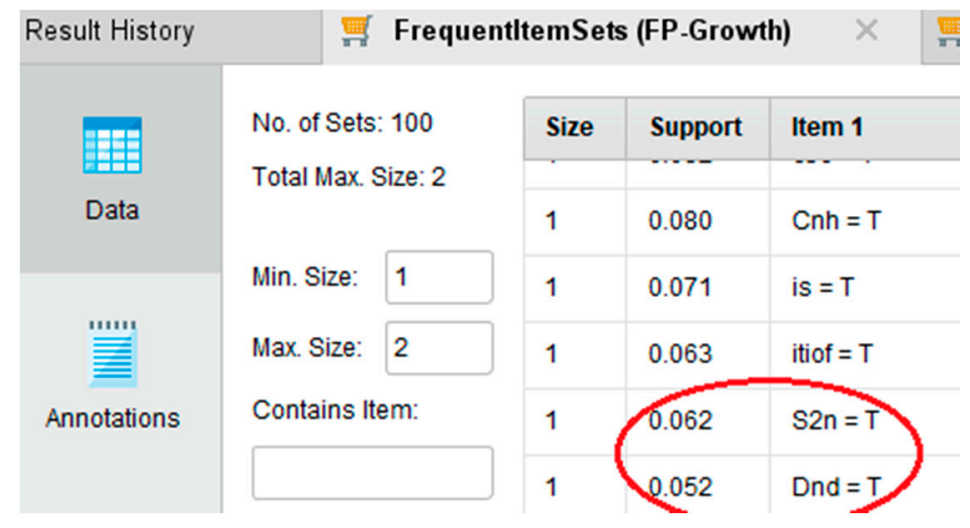

Figure 16. Support of items.

\subsubsection{Principle 4: Force Balance}

The first impression of symmetry to most people is balance and beauty. In fact, balance is indeed an important function of symmetry. Almost all the symmetry elements in Table 1 have the requirement of counteracting side effects, which includes the balance of force. There are many cases in machinery that use symmetrical structures to achieve self-balancing.

The balance of radial force mainly depends on rotational symmetry. Except for $C_{1}$, all the other point group symmetry can achieve the balance of radial force. The balance of axial force mainly depends on mirror symmetry, and point group symmetry that realize the force self-balance are $C_{S}, C_{n h}$, $D_{n h}, D_{n d}, S_{2 n}$, and $K_{h}$. As shown in Figure 17, regardless of whether the cylinder arrangement is linear or V-shaped, the engine crankshaft is mirror-symmetrical and belongs to $C_{S}$. The symmetry element $\sigma$ is located in the middle of the shaft and is perpendicular to the axis to counteract the axial forces and moments generated during the movement of the crankshaft. 


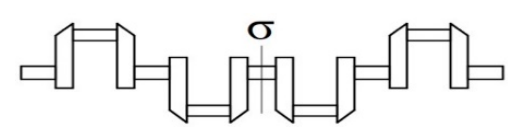

Figure 17. Crankshaft.

The improvement of air compressor is the comprehensive application of selection principle 1 , selection principle 3 and principle 4 . The working principle of a common piston air compressor is shown in Figure 18a. During operation, the motor drives the crank linkage, which drive its piston to create a linear reciprocating motion. The piston force acts on the crankshaft through the connecting rod, and the overturning moment $M_{N}$ and resisting moment $M_{y}$ are generated. The overturning moment $M_{N}$ acts on the air compressor, causing the air compressor to obviously shake. The resistance moment $M_{y}$ acts on the spindle at the center of the crank, which accelerates or decelerates the spindle, thereby causing vibration and noise in the air compressor. The lateral force $N$ of the piston pressing on the cylinder block increases their friction and accelerates the wear of the contact surface, thus affecting the sealing performance and reducing the service life of the air compressor [34]. The double crank linkage shown in Figure $18 \mathrm{~b}$ has only mirror symmetry and belongs to $C_{S}$, which means that the harmful forces cancel each other out. Therefore, the vibration and noise of the air compressor caused by the periodic change in resistance moment $M_{y}$ can be eliminated, and the friction force between the piston and cylinder block caused by the lateral force $N$ can be reduced, thus reducing the wear and prolonging the service life of the air compressor. However, that type of air compressor is single-acting, the output gas with pressure is discontinuous, and the pressure pulsation is large. Figure 18c shows an air compressor of $C_{n v}$. This layout not only counteracts harmful forces but also outputs continuous high-pressure gas and reduces pressure pulsation.

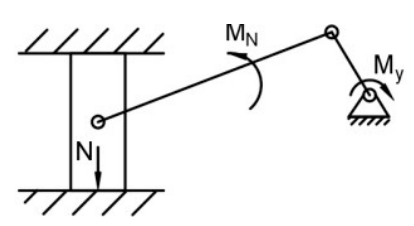

(a)

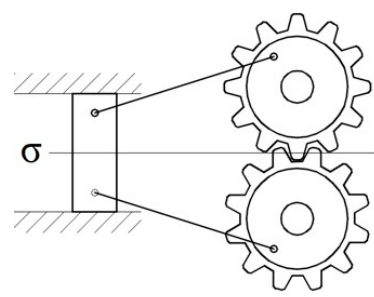

(b)

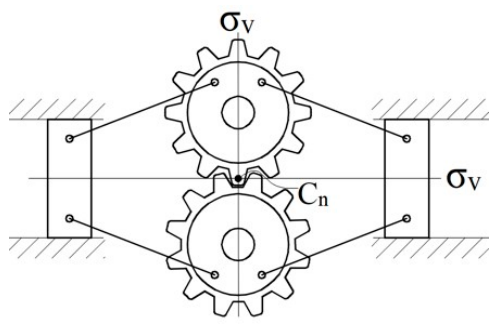

(c)

Figure 18. Crank linkage. (a) Single crank linkage; (b) double crank linkage; (c) four crank linkage.

\section{Patent}

Gear pumps are common hydraulic pumps. Generally, the inlet chamber of a pump is directly connected to the outside world, and its pressure is equal to the ambient pressure. The outlet chamber is generally connected to the actuator, and its pressure is higher than the ambient pressure. The pressure difference between inlet chamber and outlet chamber makes the gear deflect to one side, which easily leads to low efficiency and even jamming of the pump, thus reducing the service life of the gear pump. To improve the efficiency and service life of the pump, it is necessary to redesign the structure to improve the force state of the gear.

\subsection{Gear Pump of $C_{2 v}$}

In Figure 19, $F$ is the equivalent radial force acting on the gear, pointing from the high-pressure chamber to the low-pressure chamber. Because the symmetry elements of the gears in the pump are $C_{n}$ and $\sigma_{v}$, thus, the equivalent radial forces of hydraulic pressure acting on the driving and driven gears are the same. 


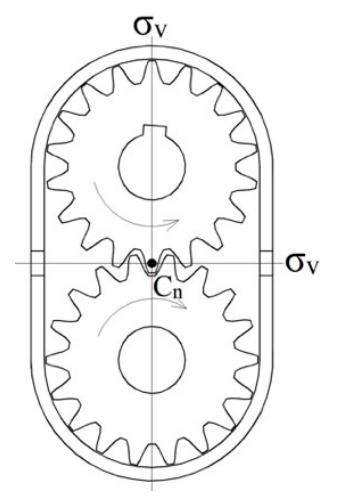

Figure 19. Gear pump of $C_{2 v}$.

\subsection{Gear Pump of $C_{4 v}$}

Figure 20 shows a four-wheel linkage gear pump [35]. After adding a pair of gears, the structure has $\sigma_{v}$ in four dimensions and $C_{n}$ as the main axis. Like the conventional gear pump, it is of $C_{n v}$, but the value of $n$ is increased. The equivalent radial force can be counteracted by this symmetrical layout, thus effectively reducing the hydraulic pressure on the gears, extending the life of the gear pump, and improving the efficiency by double suction and double output.

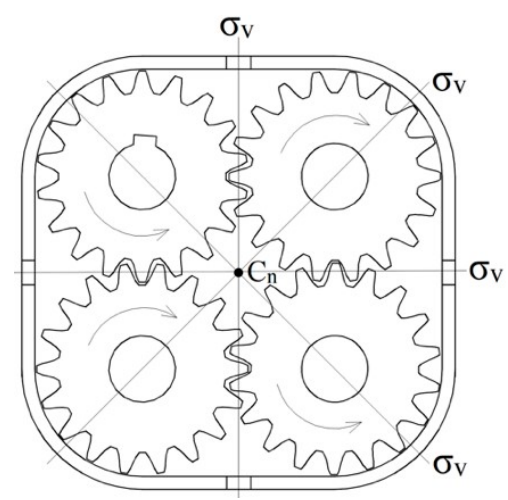

Figure 20. Gear pump of $C_{4 v}$.

The gear pump transfers oil via the gear meshing motion. In the four-wheel linkage gear pump, four gears form four pairs of gears. The transportation route of oil at the four meshing points is not clear; thus, the hydraulic pressure issue is not effectively solved. At the same time, the larger size on the plane perpendicular to the main axis will make the pump body occupy too much space.

The purpose of force balance has been defined. Firstly, the application of point group symmetry in force balance is explained through an improved piston air compressor shown in Figure 18. Then, combined with the selection principles, a new type of gear pump with radial force balancing is invented, and the authorization announcement has been obtained [36].

\subsection{Gear Pump of $D_{2 h}$}

\subsubsection{Determination of Scheme}

The gear pump of $C_{n v}$ needs to be improved to solve the problem of force imbalance, and there is no reason for asymmetry, so, according to the selection principle 1 , selection principle 3 , and selection principle 4 , the symmetry type of improved structure is determined to be $D_{n h}$. Since the structure of the original gear pump belongs to $C_{n v}$, only $\sigma_{h}$ is added to the symmetry elements of $C_{n v}$, and the final symmetry elements forming $D_{n h}$ are shown in Figure 21. 


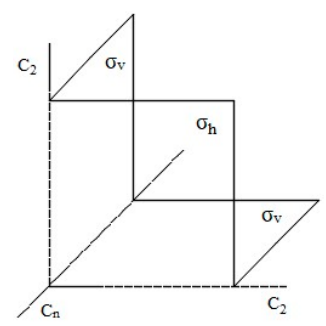

Figure 21. The spatial position of symmetry elements of $D_{n h}(n=2)$.

The structure of the gear pump improved to $D_{n h}(n=2)$ is shown in Figure 22. The size along the main axis is obviously shorter than the size perpendicular to the main axis. Two gear pairs are added along the main axis of the conventional gear pump, and the increased mirror element is $\sigma_{h}$, which can counteract additional side-effect forces. Compared with the four-wheel gear pump, the new gear pump adopts the symmetrical layout of $D_{n h}$, which has a smaller volume and a clearer oil transmission route.

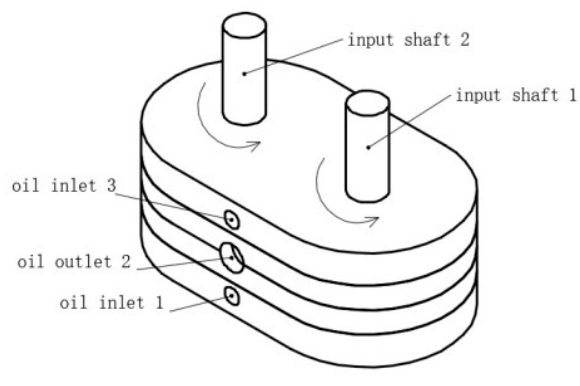

(a)

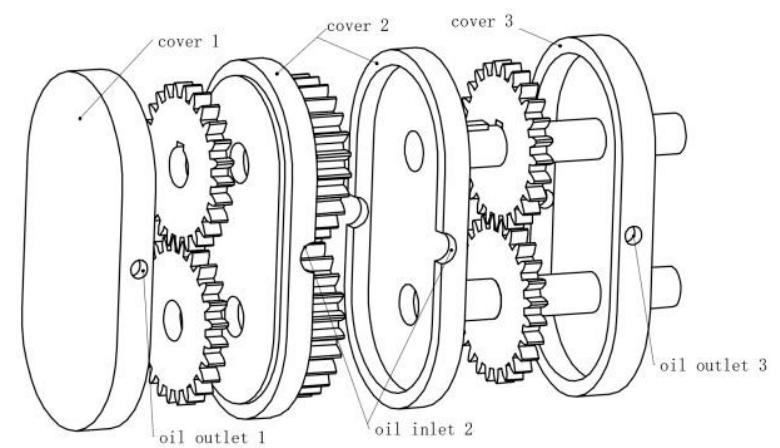

(b)

Figure 22. New gear pump. (a) Assembly; (b) exploded view.

\subsubsection{Balance of Force}

The new gear pump has three pairs of gears along the main axis, and each pair of gears has symmetry elements $\sigma_{v}$ and $C_{n}$. The radial force at each shaft is equivalent to $F_{1}, F_{2}$, and $F_{3}$, as shown in Figure 23, which is linearly related to the width of the gear. When other variables are controlled, the following conditions are established:

$$
2 B_{\text {first gear pair }}=B_{\text {second gear pair }}=2 B_{\text {third gear pair }}\left(B_{x} \text { is the width of the gear }\right)
$$

Oil inlet 1 and oil inlet 3 are located on the same side and on a different side from oil inlet 2 . The radial force is directed from the outlet chamber to the inlet chamber such that $F_{1}$ and $F_{3}$ point to the same direction, opposite to $F_{2}$.

Thus, the following equation can be obtained:

$$
2 F_{1}=F_{2}=2 F_{3}
$$

The forces and moments on the input shaft 1 or input shaft 2 are shown in Figure 23, and $M_{1}$ and $M_{2}$ are bending couples generated by radial forces. Because of $\sigma_{h}, M_{1}$ and $M_{2}$ are of the same size and opposite directions and can cancel each other out. 


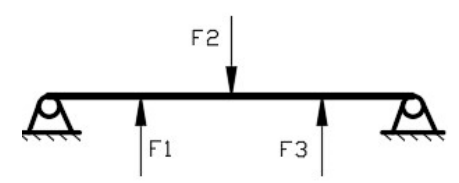

(a)

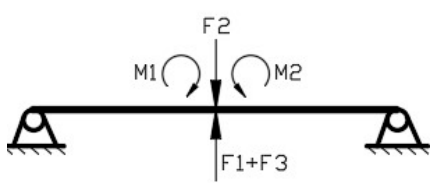

(b)

Figure 23. Force analysis. (a) Radial force on the input shafts; (b) force addition.

According to the selection principles, the symmetry type of gear pump is changed from $C_{n v}$ to $D_{n h}$. Through the force analysis, it is proved that the layout of $D_{n h}$ can balance the side effects generated during the gear operation. The high-pressure oil delivered out of three oil outlets can be applied to one working mechanism or to three working mechanisms at the same time. When the oil is applied to three working mechanisms, in addition to counteracting the radial force, the design of $D_{n h}$ can also meet the needs of synchronous operation of different mechanisms.

\section{Conclusions}

In this paper, the idea of group theory was introduced into mechanical structure design, and the research started from the point group symmetry, and the following progress was made:

1. Based on the definition and symbol of crystal point group symmetry and the characteristics of machinery, the concept system of the point group symmetry of mechanical structures was proposed.

2. The requirements of symmetry elements were summarized, and the corresponding requirements of point group symmetry could be obtained through the combination of symmetry elements.

3. By using the data mining software RapidMiner, the association rules between point group symmetry and requirements were mined. The results of mining could be used as a reference for structural improvement or innovation.

4. Four selection principles for point group symmetry were proposed. Compared with a large number of mining results, the selection principles could provide a faster design idea.

5. Based on the selection principles and the structural characteristics, a new gear pump with radial force balancing was invented.

This paper only discusses the point group symmetry of mechanical structures. The application of space group symmetry and group theory in micromechanical systems, such as dynamic symmetry, is the subject of future research. In addition, it is more important to refine the regular knowledge related to symmetry and to use symmetry as a method of design.

Author Contributions: Writing-review and editing, X.C.; methodology, X.C. and P.F.; project administration, Q.Q.; supervision, C.Y.; funding acquisition, Q.Q. and P.F. All authors have read and agreed to the published version of the manuscript.

Funding: This research was funded by [National Natural Science Foundation of China] grant number [51175455] and [Key Research and Development Plan Project of Zhejiang Province] grant number [2019C01053].

Conflicts of Interest: The authors declare no conflict of interest.

\section{References}

1. Rosen, J. Symmetry Rules: How Science and Nature Are Founded on Symmetry; Springer: Berlin, Germany, 2008; pp. 1-15.

2. Sabelli, H.; Lawandow, A.; Kopra, A. Asymmetry, symmetry and beauty. Symmetry 2010, 2, $1591-1624$. [CrossRef]

3. Whalen, A.J.; Brennan, S.N.; Sauer, T.D. Observability and Controllability of Nonlinear Networks: The Role of Symmetry. Phys. Rev. X 2015, 5, 1. [CrossRef]

4. Trost, B.M.; Bunt, R.C. On the Question of the Symmetry of Formally Symmetrical $\pi$-(Allyl) palladium Cationic Intermediates in Allylic Alkylations. J. Am. Chem. Soc. 2016, 118, 235-236. [CrossRef] 
5. Balaji, S. Internal symmetry in protein structures: Prevalence, functional relevance and evolution. Curr. Opin. Struc. Biol. 2015, 32, 156-166. [CrossRef] [PubMed]

6. Nimmo, R. Animal Cultures, Subjectivity, and Knowledge: Symmetrical Reflections beyond the Great Divide. Soc. Anim. 2012, 20, 173-192. [CrossRef]

7. Montoya, F.G.; Baños, R.; Alcayde, A.; Manzano-Agugliaro, F. Symmetry in Engineering Sciences. Symmetry 2019, 11, 797. [CrossRef]

8. Andersson, S.A.; Crawford, J.B. General canonical correlations with applications to group symmetry models. J. Multivar. Anal. 2010, 101, 1547-1558. [CrossRef]

9. Everett, L.L.; Stuart, A.J. The Double Cover of the Icosahedral Symmetry Group and Quark Mass Textures. Phys. Lett. B 2011, 698, 131-139. [CrossRef]

10. Zingoni, A. Group-theoretic exploitations of symmetry in computational solid and structural mechanics. Int. J. Meth. Eng. 2010, 79, 253-289. [CrossRef]

11. Stewart; James, J.P. Symmetry groups for unit cells in solids. J. Comput. Chem. 2015, 19, 168-180.

12. Gelashvili, D.B.; Chuprunov, E.V.; Marychev, M.O. The application of group theory to the description of pseudosymmetry. Biol. Bull. 2011, 1, 185-198. [CrossRef]

13. Leyton, M. Group Theory and Architecture. Nexus Netw. J. 2001, 3, 39-58. [CrossRef]

14. Lukashchuk, S.Y.; Saburova, R.D. Approximate symmetry group classification for a nonlinear fractional filtration equation of diffusion-wave type. Nonlinear Dynam. 2018, 93, 295-305. [CrossRef]

15. Pahl, G.; Beitz, W.; Feldhusen, J.; Grote, K.H. Engineering Design: A Systematia Approach; Springer: Berlin, Germany, 2007.

16. Sun, N.P. Axiomatic Design: Advanced and Applications; Oxford University Press: Oxford, UK, 1998.

17. Altshuller, G.S. Creativity as an Exact Science: The Theory of Inventive Problem Solving; Gordon and Breach Publisher: New York, NY, USA, 1988.

18. Barrenscheen, J. Systematic Exploitation of Symmetrical Properties in Construction. Ph.D. Thesis, Technical University of Braunschweig, Braunschweig, Germany, 1990.

19. Li, M.; Langbein, F.C.; Martin, R.R. Detecting design intent in approximate CAD models using symmetry. Comput. Aided Des. 2010, 42, 183-201. [CrossRef]

20. Zheng, Y.; Zhang, L.; Xiao, T. Symmetry detection algorithm in design for assembly. J. Tsinghua Univ. 2008, 48,490 .

21. Noga, S.; Bogacz, R.; Frischmuth, K. Vibration analysis of toothed gear with cyclic symmetry modelling. Vib. Phys. Syst. 2012, 25, 299-304.

22. Wang, Y.; Xu, K.; Li, J. Symmetry Hierarchy of Man-Made Objects. Comput. Graph. Forum 2011, 30, $287-296$. [CrossRef]

23. Gesu, V.D.; Tabacchi, M.E.; Zavidovique, B. Symmetry as an Intrinsically Dynamic Feature. Symmetry 2010, 2, 554-581. [CrossRef]

24. Ren, J.; Jiang, X.; Yuan, J. LBP-Structure Optimization with Symmetry and Uniformity Regularizations for Scene Classification. IEEE Signal. Proc. Lett. 2016, 24, 37-41. [CrossRef]

25. Várkonyi, P.L.; Domokos, G. Symmetry, optima and bifurcations in structural design. Nonlinear Dynam. 2006, 43, 47-58. [CrossRef]

26. Qiu, Q.Y.; Qi, Y.X.; Feng, P.E. Assembly efficiency prediction model of parts based on multi symmetry features. J. Mech. Eng. 2017, 53, 118-124. [CrossRef]

27. Gu, C.S.; Sun, Y.C.; Yang, C.J.; Cao, H.J. Novel Tailgate Limiting Stopper Assembly. Chinese Ptatent 201510332118.9, 1 March 2017.

28. Müller, U. Symmetry Relationships between Crystal Structures: Applications of Crystallographic Group Theory in Crystal Chemistry; Oxford University Press: Oxford, UK, 2013; p. 67.

29. Li, X.C.; Li, X.B. Reasonable Design Atlas of Mechanical Structure; Shanghai Scientific \& Technical Publishers: Shanghai, China, 1996; p. 10.

30. Yao, Z.F.; Wang, F.J.; Yang, M.; Qu, L.X.; Xiao, R.F.; Xu, X.D.; He, C.L. Effects of Impeller Type on Pressure Fluctuations in Double-suction Centrifugal Pump. J. Mech. Eng. 2011, 47, 133-137. [CrossRef]

31. Zhang, X.H.; Deng, P.F.; Luo, W.G. Multidirectional Vibration Energy Collection Device with Replaceable Energy Collection Module. Chinese Ptatent 201520704388.3, 13 January 2016.

32. Kotu, V.; Deshpande, B. Predictive Analytics and Data Mining: Concepts and Practice with Rapidminer; Elsevier: Singapore, 2014; p. 163. 
33. Yang, Z.Q.; Fei, Y.Z.; Liu, H.G. Asymmetrical Lifting Traverse. Chinese Ptatent 201510481201.2, 8 March 2017.

34. Li, Z.W.; Sheng, X.M. Double-acting air-oil intensifier driven by twin roller piston air cylinder. Appl. Mech. Mater. 2012, 220-223, 580-584. [CrossRef]

35. Chang, Y.; Zhu, S.S.; Hu, M.W.; Guo, X.L.; Yang, Y.C. A Gear Pump with Radial Force Balance. Chinese Ptatent 201510962801.0, 27 June 2017.

36. Qiu, Q.Y.; Chen, X.M.; Feng, P.E.; Yang, C. A Multi-Wheel Linkage Gear Pump with Radial Force Balance. Chinese Ptatent 201920869252.6, 28 February 2020.

(C) 2020 by the authors. Licensee MDPI, Basel, Switzerland. This article is an open access article distributed under the terms and conditions of the Creative Commons Attribution (CC BY) license (http://creativecommons.org/licenses/by/4.0/). 